\title{
Retrospective cohort of 24,974 surgical cases under spinal anesthesia: Bupivacaine dose and failure rate
}

Alexander Avidan, MD, Charles Weissman, MD, Carolyn F. Weiniger, MD

Department of Anesthesiology and Critical Care Medicine, Hadassah - Hebrew University Medical Center, Jerusalem Israel.

\section{Background and Goals}

Per text books and current literature, bupivacaine is the preferred local anesthetic for spinal anesthesia. Recommended doses range from low (up to $5 \mathrm{mg}$ ) to high (up to 15-20mg). There is a debate about the selection of hyperbaric, hypobaric or plain bupivacaine. The goal of this study was to investigate the selected bupivacaine dose used for spinal anesthesia for common lower body surgery and to examine the failure rates.

\section{Material and Methods}

This study was approved by the Hadassah Institutional Review Board (IRB). Data from anesthetics with spinal anesthesia for various types of surgery of the lower body (obstetric, gynecologic, proctologic, orthopedic, urologic, inguinal hernia and bone marrow aspiration) were retrieved from the anesthesia information management system (Metavision, iMDsoft, Israel) of the Hadassah - Hebrew University Medical Center from February 2007 to December 2016.

\section{Results and Discussion}

Data from 24,974 anesthesia records were retrieved and are summarized in Table 1. Hyperbaric bupivacaine was the most frequent choice for all surgeries, and most frequently reported sensory levels were appropriate to cover for the surgical innervation. The sensory level was not reported in $57 \%$ of cases. Overall, $2.2 \%$ of the spinals were reported as failures.

\section{Table 1}

\begin{tabular}{|c|c|c|c|c|c|c|c|c|c|c|}
\hline \multirow[b]{3}{*}{$\begin{array}{l}\text { Type of } \\
\text { Surgery }\end{array}$} & \multirow[b]{3}{*}{$\begin{array}{l}\mathrm{N} \\
\% \\
\end{array}$} & \multicolumn{2}{|c|}{ Duration (minutes) } & \multicolumn{6}{|c|}{ Dose Bupivacaine (mg) } & \multirow{3}{*}{$\begin{array}{c}\text { Failure Rate } \\
\qquad \mathrm{N}(\%)\end{array}$} \\
\hline & & \multirow{2}{*}{$\begin{array}{c}\text { Anesthesia } \\
\text { Mean SD }\end{array}$} & \multirow{2}{*}{$\begin{array}{l}\text { Surgery } \\
\text { Mean SD }\end{array}$} & \multicolumn{2}{|c|}{ Hyperbaric } & \multicolumn{2}{|c|}{ Hyporbaric } & \multicolumn{2}{|c|}{ Plain } & \\
\hline & & & & $\mathrm{N}(\%)$ & Mean SD & $\mathrm{N}(\%)$ & Mean, SD & $\mathrm{N} \%$ & Mean SD & \\
\hline Orthopedic & $9742(39.0 \%)$ & $100 \pm 178$ & $59 \pm 173$ & $7613(82.2 \%$ & $9.2 \pm 2.9$ & $756(8.2 \%)$ & $9.4 \pm 4.1$ & $820(8.9 \%)$ & $6.6 \pm 3.6$ & $205(2.1 \%)$ \\
\hline $\begin{array}{c}\text { Cesarean } \\
\text { delivery }\end{array}$ & $8814(35.3 \%)$ & $73 \pm 165$ & $31 \pm 23$ & 7937 (92.9\%) & $9.0 \pm 2.3$ & $13(0.2 \%)$ & $9.2 \pm 1.9$ & $598(7.0 \%)$ & $7.9 \pm 3.3$ & $147(1.7 \%)$ \\
\hline $\begin{array}{l}\text { Inguinal hernia } \\
\text { repair }\end{array}$ & $1886(7.6 \%)$ & $76 \pm 55$ & $48 \pm 22$ & $1703(93.1 \%)$ & $9.6 \pm 4.1$ & $9(0.5 \%)$ & $9.1 \pm 1.5$ & $118(6.4 \%)$ & $7.8 \pm 1.8$ & $98(5.2 \%)$ \\
\hline Urology & $2253(9.0 \%)$ & $73 \pm 43$ & $43 \pm 26$ & $2105(97.1 \%)$ & $9.6 \pm 2.6$ & $5(0.2 \%)$ & $8.8 \pm 1.1$ & $57(2.6 \%)$ & $8.2 \pm 1.8$ & $68(3.0 \%)$ \\
\hline Proctology & $1701(6.8 \%)$ & $49 \pm 110$ & $19 \pm 12$ & $1590(98.7 \%)$ & $8.5 \pm 2.3$ & $4(0.2 \%)$ & $7.9 \pm 1.7$ & $17(1.1 \%)$ & $7.7 \pm 1.8$ & $21(1.2 \%)$ \\
\hline Gynecology & $508(2.0 \%)$ & $63 \pm 25$ & $37 \pm 20$ & 458 (96.2\%) & $8.6 \pm 2.0$ & 0 & & $18(3.8 \%)$ & $9.0 \pm 2.6$ & $15(3.0 \%)$ \\
\hline $\begin{array}{l}\text { Bone marrow } \\
\text { aspiration }\end{array}$ & $70(0.3 \%)$ & $85 \pm 28$ & $45 \pm 22$ & $67(95.7 \%)$ & $10.2 \pm 1.7$ & 0 & & $3(4.3 \%)$ & $8.3 \pm 3.2$ & $6(8.6 \%)$ \\
\hline Total & 24974 & $74 \pm 35$ & $42 \pm 26$ & $21473(98.7 \%)$ & $9.1 \pm 2.7$ & $787(0.2 \%)$ & $9.4 \pm 4.0$ & $1631(1.1 \%)$ & $7.2 \pm 3.4$ & $560(2.2 \%)$ \\
\hline
\end{tabular}

\section{Conclusions}

The selection and dose of spinal anesthesia were suitable for the duration of surgery. Hyperbaric bupivacaine was the most common choice. Doses were between the recommended low and high doses. The failure rate was like that reported in the literature. 